Chinese Journal of Organic Chemistry

\title{
一类新型大环内酯衍生物的合成及其生物活性研究
}

\author{
史大昕 ${ }^{a}$ 冯 雪 ${ }^{a}$ 庄晓磊 ${ }^{a}$ 柴洪新 ${ }^{a}$ 刘 霆 ${ }^{b}$ \\ 张 奇*,a 李加荣*, \\ $\left({ }^{a}\right.$ 北京理工大学化工与环境学院 北京 100081) \\ $\left(^{b}\right.$ 北京市农林科学研究院 北京 100097)
}

\begin{abstract}
摘要 为了寻找高效、广谱的杀虫、杀菌剂，以去糖基或部分去糖基的多杀菌素为母体进行结构修饰，合成了 8 种大 环内酯酰化衍生物, 其结构经 ${ }^{1} \mathrm{H} \mathrm{NMR},{ }^{13} \mathrm{C} \mathrm{NMR}$ 和 MS 表征. 对合成的化合物进行了生物活性测试. 结果表明, 这类化 合物对鳞翅目、鞘翅目、双翅目、半翅目及线虫等害虫有较好的杀灭活性, 其中化合物 $3 \mathrm{e}$ 在 $100 \mathrm{mg} / \mathrm{L}$ 浓度下对桃蚜 的 $24 \mathrm{~h}$ 杀灭率达到 $100 \%$, 化合物 $\mathbf{3 f}$ 和 $\mathbf{3 h}$ 在上药量为 $200 \mu \mathrm{g}$ 时对假单胞菌有一定的抑制作用.
\end{abstract}

关键词 大环内酯; 酰化; 杀虫活性; 杀菌活性

\section{Study of Synthesis and Biological Activities of a Novel Macrolide Derivatives}

\author{
Shi, Daxin ${ }^{a} \quad$ Feng, Xue $^{a} \quad$ Zhuang, Xiaolei $^{a} \quad$ Chai, Hongxin $^{a}$ \\ Liu, Ting ${ }^{b}$ Zhang, Qi ${ }^{*, a} \quad$ Li, Jiarong*,a \\ ( ${ }^{a}$ School of Chemical Engineering and Environment, Beijing institute of Technology, Beijing 100081) \\ ( ${ }^{b}$ Beijing Academy of Agricultural and Forestry Sciences, Beijing 100097)
}

\begin{abstract}
Modification of molecular structure of pesticides is a common method to seek new efficient broad-spectrum insecticides. This study focused on modifying the structure of deglycosylated or partially deglycosylated spinosad. Eight new macrolide derivatives had been synthesized by acylation. Their structures were characterized by ${ }^{1} \mathrm{H} N M R,{ }^{13} \mathrm{C}$ NMR and MS techniques. Their fungicidal and insecticidal activities were also evaluated. The results showed that all compounds synthesized had excellent insecticidal activities against lepidoptera, coleoptera, diptera, hemiptera and nematoda. Compound $\mathbf{3 e}$ at the concentration of $100 \mathrm{mg} / \mathrm{L}$ presented $100 \%$ insecticidal activity against hemiptera (e.g. Myzus persicae) and compounds $\mathbf{3 f}$ and $\mathbf{3 h}$ loading $200 \mu \mathrm{g}$ presented certain inhibition against pseudomonas.

Keywords macrolide; acylate; insecticidal activity; fungicidal activity
\end{abstract}

大环内酯抗生素是一类在医药和农药领域有着广 泛应用的抗菌药物 ${ }^{[1 \sim 3]}$, 已被列入世界优先发展的十类 药物之一 ${ }^{[4]}$. 自 1952 年开发第一代大环内酯抗生素红霉 素至今共有三代大环内酯抗生素, 其中第三代大环内酯 类抗生素主要包括酮内酯 $[5,6]$ 、酰内酯、烯内酯等 ${ }^{[6,7]}$. 其 代表性药物有泰利霉素和赛红霉素, 这两个药物均具有 较好的应用前景 ${ }^{[8-10]}$. 第三代大环内酯除了在医药中具 有非常好的应用效果外, 在农药方面也有突出的杀虫杀 螨活性, 典型的例子有多杀菌素和乙基多杀菌素. 它们 都是性能优异的绿色杀虫剂 ${ }^{[11 ~ 14]}$, 具有低毒、低残留、
对天敌安全、对环境无毒害、分解快等优点 ${ }^{[15 ~ 19]}$, 兼具 生物农药的安全性和化学合成农药的速效性.

对大环内酯的结构修饰是目前国内外的研究重 点 ${ }^{[20 ~ 22]}$, 主要涉及大环内酯母体结构的修饰、侧链糖基 的修饰以及所连糖基的替换等 ${ }^{[20,21]}$.

考虑到大环内酯分子中引入酰基能产生如下有利 影响，即降低前体的挥发性、延长作用时间、增加母环 的光稳定性，同时能改善母环的亲脂性，使大环内酯更 易进入害虫体内, 增加其杀虫活性. 因此, 本研究利用 多杀菌素降解产生的大环内酯作为主体骨架，在原糖基

\footnotetext{
*E-mail: zhangqi@bit.edu.cn,jrli@bit.edu.cn

Received May 6, 2014; revised July 28, 2014; published online August 11, 2014

Project supported by the Basic Research Fund of Beijing Institute of Technology (No. 20131042006).

北京理工大学基础研究基金(No. 20131042006)资助项目.
} 
所在的羟基位置进行酰化改造; 并研究衍生化产物的杀 虫、杀菌活性, 以期获得活性更高、杀虫谱更广、环境 兼容性更好的新化合物.

\section{1 结果与讨论}

\section{1 合成设计}

本文以多杀菌素的降解产物(I 或 II)为原料, 通过 对大环内酯环上的羟基进行酰化反应，合成了 8 种新型 大环内酯衍生物 $\mathbf{3 a} \sim \mathbf{3 h}$ (Scheme 1); 通过 ${ }^{1} \mathrm{H}$ NMR, ${ }^{13} \mathrm{C}$ NMR 和质谱等表征确定了新合成产物的结构.

\section{2 反应条件选择}

考虑到大环内酯的结构不稳定, 而酰化反应又显著 放热, 因此加入酰化试剂时选择较低温度(如需要可用 冰浴降温), 待反应进行一段时间温度稳定后, 可适当 升温以加速反应的进行. 研究主要采用 DMAP 作催化 剂, 催化效果较好(表 1). 但由于大环内酯的体积较大, 如果酰化试剂位阻也较大，则酰化反应较难进行; 特别 是母环骨架上需要两个羟基同时酰化, 则需要更强的催
化剂. 如选用金刚烷甲酰氯酰化时，需要 $\mathrm{NaH}$ 催化，问 题是此时反应液变得浑浊, 有可能是醇钠盐的产生降低 了反应物的溶解度所致, 因此需要更长的反应时间. 由 于大环内酯底物的体积较大, 因此酰化时主要考虑酰化 试剂的空间位阻和亲电性两方面的影响, 酰化试剂的位 阻越大, 亲电性越弱, 反应的产率越低; 如位阻较大的 金刚烷甲酰氯作为酰化试剂时, 产物 $3 \mathrm{e}$ 和 $3 \mathrm{~h}$ 的产率相 对较低; 缺电子的 3-吡啶甲酰氯的酰化产物产率较高.

\section{3 产物结构表征}

${ }^{1} \mathrm{H}$ NMR 谱图中大环内酯母环上饱和氢的化学位移 在 $\delta 1.00 \sim 4.00$, 双键氢的化学位移分别在 $\delta 5.90$ 和 6.80 左右. ${ }^{13} \mathrm{C}$ NMR 谱图中大环内酯母环上饱和碳的化学位 移在 $\delta 10.00 \sim 50.00$, 双键碳的化学位移在 $\delta 130.00 \sim$ 145.00 左右, 羰基碳在 $\delta \quad 170.00 \sim 200.00$ 左右. 酰化反 应后引入的基团上的质子和碳谱的信号峰与大环内酯 自身的信号峰区分明显. 以化合物 $\mathbf{3 f}$ 为例, ${ }^{1} \mathrm{H}$ NMR 谱 图中化学位移在 $\delta 2.05$ 处的单峰为大环内醌衍生物 17乙酰基上的氢质子, $\delta 2.02$ 处的单峰为大环内酯衍生物

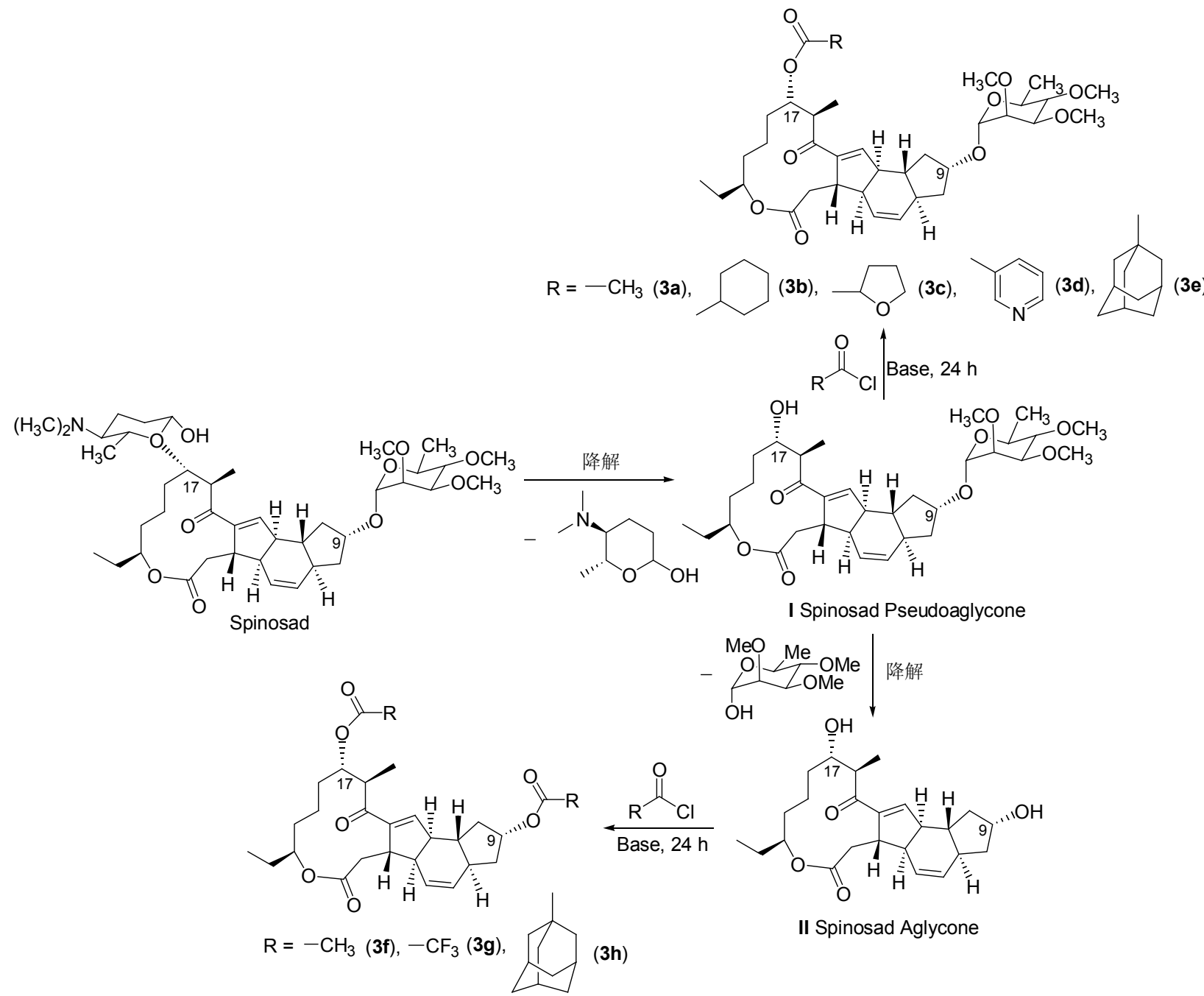

Scheme 1 
表 1 不同催化剂对反应产物的影响

Table 1 The effect of different catalysts on synthetic reaction

\begin{tabular}{|c|c|c|c|}
\hline Compd. & Stucture & Catalyst & Yield \\
\hline $3 a$ & & DMAP & $95 \%$ \\
\hline $\mathbf{3 b}$ & & DMAP & $80 \%$ \\
\hline $3 c$ & & DMAP & $85 \%$ \\
\hline 3d & & DMAP & $86 \%$ \\
\hline $3 e$ & & DMAP & $80 \%$ \\
\hline $3 f$ & & DMAP & $95 \%$ \\
\hline
\end{tabular}




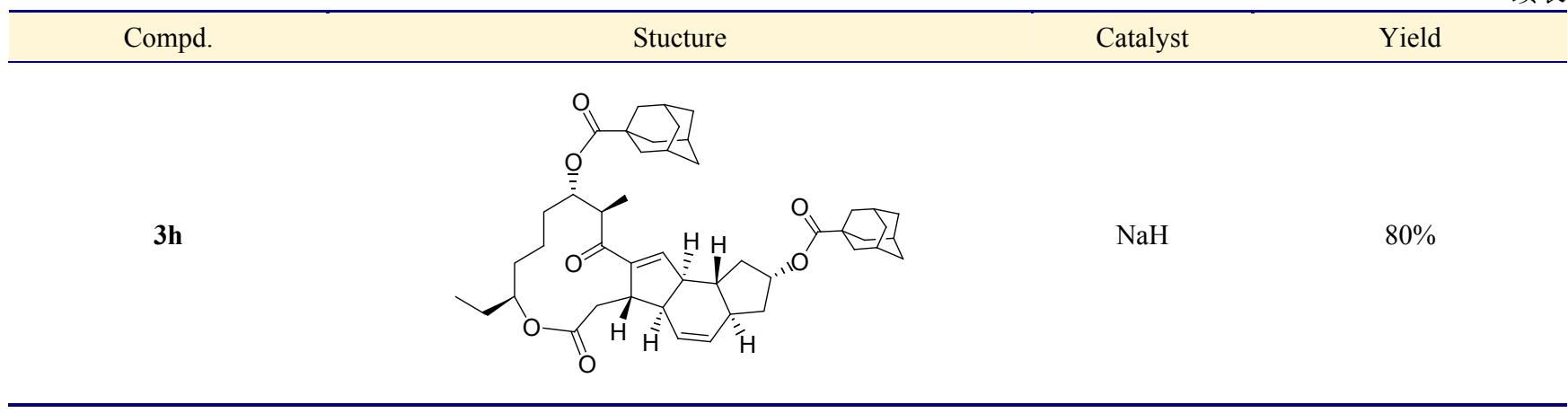

9-乙酰基上的氢质子; ${ }^{13} \mathrm{C} \mathrm{NMR}$ 谱图中化学位移 $\delta$ 170.82 和 170.47 处为乙酰基的羰基碳，化学位移 $\delta 21.29$ 和 21.05 处为乙酰基的甲基碳. 核磁数据表明在大环内 酯环上成功引入乙酰基. 在 MALDI TOF 质谱中通常出 现目标化合物的 $[\mathrm{M}+\mathrm{Na}]^{+}$峰.

\section{4 生物活性}

对所合成的大环内酯衍生物进行了杀虫、杀菌活性 测试，其中杀虫活性采用的测试标本有鳞翅目、鞘翅目、 双翅目、半翅目害虫及线虫; 杀菌活性测试则采用了假 单胞菌. 测定结果显示, 所有大环内酯衍生物物均有不 同程度的杀虫杀菌活性. 在所测试的大环内酯衍生物 中, 具有直链烷基和杂环取代的化合物对小菜夜蛾的杀 虫活性较好. 浓度为 $100 \mathrm{mg} / \mathrm{L}$ 时，化合物 3a, 3e, 3f, 3g 均具有较高的杀虫率 $(24 \mathrm{~h})$. 对桃蚜虫的杀虫活性研究 显示: 浓度为 $100 \mathrm{mg} / \mathrm{L}$ 时, 化合物 $3 \mathrm{e}$ 的 $24 \mathrm{~h}$ 杀虫率约 为 $100 \%$, 化合物 $3 \mathrm{c}$ 的 $24 \mathrm{~h}$ 杀虫率大于 $50 \%$. 对秀丽线 虫而言: 杂环取代的大环内酯衍生物具有较好的活性, 其 $24 \mathrm{~h}$ 内对虫子有明显抑制作用, $48 \mathrm{~h}$ 内有杀虫活性. 杀菌活性的测定结果显示: 化合物 3b, 3c, 3d, 3f, 3h 对 假单胞菌的生长都有一定的抑制作用.

活性研究结果表明，用金刚烷甲酰基和四氢糠酰基 修饰后的大环内酯, 都表现出较好的杀虫杀菌活性. 其 中用四氢糠酰基修饰的衍生物有可能是提高了其与害 虫体内含糖物质的相容性, 使得害虫的排异降低, 延长 药物在虫子体内的作用时间, 改进了杀虫效果. 金刚烷 甲酰基也作为一类活性物质的改性基才，但文献报道的 修饰物一般仅有杀菌活性 ${ }^{[23]}$; 而本研究的大环内酯的 金刚烷甲酰基衍生物可以同时表现出较好的杀虫杀菌 活性.

\section{2 结论}

以多杀菌素降解产物为原料, 设计并研究了其酰化 反应, 确定了酰化的较优条件是: $40 \sim 60{ }^{\circ} \mathrm{C}$, DMAP 或 $\mathrm{NaH}$ 催化. 合成了 8 种未见文献报道的大环内酯衍生物,
所合成化合物的结构通过 ${ }^{1} \mathrm{H} N \mathrm{NM} 、{ }^{13} \mathrm{C} \mathrm{NMR}$ 和 MS 表 征确定. 对所合成的大环内酯衍生物进行了杀虫、杀菌 活性测试，结果表明 8 种化合物对鳞翅目、鞘翅目、双 翅目、半翅目害虫具有较好的杀虫活性，其中化合物 $3 \mathrm{e}$ 对半翅目害虫桃蚜的杀虫率达到 100\%; 化合物 $\mathbf{3 c}$ 具有 一定的线虫抑制作用. 化合物 $3 \mathbf{f}$ 和 $3 \mathbf{h}$ 对假单胞菌有一 定的抑制作用.

\section{3 实验部分}

\section{1 仪器与试剂}

本研究所用大环内酯降解物 I 和 II 按照文献[22]和 [23]报道的方法制得. 其余试剂均为市售分析纯或化学 纯商品, 未进一步纯化. 熔点测定使用 XT4 显微熔点测 定仪 (未较正); 红外光谱用 Thermo Fisher Scientific Nicolet iS10 型傅里叶变换型红外仪( $\mathrm{KBr}$ 压片测定); 核 磁共振氢谱和碳谱采用 Varian mercury-plus 400 型和 Avance DRX 500 型核磁共振仪记录, $\mathrm{CDCl}_{3}$ 为溶剂, TMS 为内标; 质谱采用 Autoflex III 型 MALDI TOF 质谱 仪测定; 高分辨质谱采用 Bruker Apex FTMS 和 Bruker Daltonics Inc. APEXII 型 FT-ICR 质谱仪.

\section{2 大环内酯衍生物的合成}

3.2.1 (2R,3aS, $5 a R, 5 b S, 9 S, 13 S, 14 R, 16 a S, 16 b R)-9$ - 乙 基-14-甲基-7,15-二羰基-2-(( $(2 R, 3 R, 4 R, 5 S, 6 S)-3,4,5$-三 甲氧基-6-甲基四氢- $2 H$-吡喃-2-) 氧代)-2,3,3a,5a,5b, $6,7,9,10,11,12,13,14,15,16 a, 16 b$-十六氢- $1 H-a s$-引达省 并[3,2-d][1]氧杂十二元环-13-乙酸酯(3a)的合成

氩气保护下, 加入多杀菌素降解产物 I $(0.3 \mathrm{~g} 0.5$ mmol)、DMAP (0.305 g, $2.5 \mathrm{mmol})$ 、无水氯仿 $(10 \mathrm{~mL})$ 、 无水乙酸乙酯 $(5 \mathrm{~mL})$, 搅拌至全部溶解. 用注射器缓慢 加入乙酸酐 $(3 \mathrm{~mL}, 2.5 \mathrm{mmol})$, 回流反应 $24 \mathrm{~h}, \mathrm{TLC}$ 监测 反应. 反应结束后依次用 $5 \% \mathrm{NaHCO}_{3}$ 溶液、饱和 $\mathrm{NaCl}$ 溶液洗涤, 合并有机相. 有机相用无水 $\mathrm{Na}_{2} \mathrm{SO}_{4}$ 干燥, 柱 层析分离, 展开剂为 $V$ (石油醚) $: V($ 乙酸乙酯 $)=3: 1$ $\left(R_{\mathrm{f}}=0.27\right)$. 旋干溶剂得黄色油状液体. ${ }^{1} \mathrm{H}$ NMR (400 
$\left.\mathrm{MHz}, \mathrm{CDCl}_{3}\right) \delta: 6.81\left(\mathrm{~s}, 1 \mathrm{H}, \mathrm{C}_{13}-\mathrm{H}\right), 5.92 \sim 5.90(\mathrm{~m}, 1 \mathrm{H}$, $\left.\mathrm{C}_{6}-\mathrm{H}\right), 5.84 \sim 5.81\left(\mathrm{~m}, 1 \mathrm{H}, \mathrm{C}_{5}-\mathrm{H}\right), 4.88\left(\mathrm{~s}, 1 \mathrm{H}, \mathrm{C}_{1^{-}-\mathrm{H}}\right)$, $4.72 \sim 4.70\left(\mathrm{~m}, 1 \mathrm{H}, \mathrm{C}_{21}-\mathrm{H}\right), 4.36 \sim 4.31\left(\mathrm{~m}, 1 \mathrm{H}, \mathrm{C}_{9}-\mathrm{H}\right)$, $3.69 \sim 3.62\left(\mathrm{~m}, 1 \mathrm{H}, \mathrm{C}_{17}-\mathrm{H}\right), 3.62 \sim 3.60\left(\mathrm{~m}, 1 \mathrm{H}, \mathrm{C}_{2^{\prime}}-\mathrm{H}\right)$, $3.59\left(\mathrm{~s}, 3 \mathrm{H}, \mathrm{C}_{4^{\prime}}-\mathrm{OCH}_{3}\right), 3.55\left(\mathrm{~s}, 3 \mathrm{H}, \mathrm{C}_{2^{\prime}}-\mathrm{OCH}_{3}\right), 3.51(\mathrm{~s}$, $\left.3 \mathrm{H}, \mathrm{C}_{3},-\mathrm{OCH}_{3}\right), 3.50 \sim 3.48\left(\mathrm{~m}, 2 \mathrm{H}, \mathrm{C}_{4}-\mathrm{H}, \mathrm{C}_{5^{\prime}}-\mathrm{H}\right), 3.42(\mathrm{dd}$, $\left.J=9.4,3.3 \mathrm{~Hz}, 1 \mathrm{H}, \mathrm{C}_{3^{\prime}}-\mathrm{H}\right), 3.18 \sim 3.16\left(\mathrm{~m}, 1 \mathrm{H}, \mathrm{C}_{16}-\mathrm{H}\right)$, $3.14 \sim 3.13\left(\mathrm{~m}, 2 \mathrm{H}\right.$, one of $\left.\mathrm{C}_{2}-\mathrm{H}, \mathrm{C}_{4^{-}}-\mathrm{H}\right), 3.08 \sim 3.05(\mathrm{~m}$, $\left.1 \mathrm{H}, \mathrm{C}_{3}-\mathrm{H}\right), 2.93 \sim 2.89\left(\mathrm{~m}, 1 \mathrm{H}, \mathrm{C}_{12}-\mathrm{H}\right), 2.44(\mathrm{dd}, J=8.0$, $2.0 \mathrm{~Hz}, 1 \mathrm{H}$, one of $\left.\mathrm{C}_{2}-\mathrm{H}\right), 2.39 \sim 2.38(\mathrm{~m}, 1 \mathrm{H}$, one of $\left.\mathrm{C}_{10}-\mathrm{H}\right), 2.30 \sim 2.26\left(\mathrm{~m}, 1 \mathrm{H}, \mathrm{C}_{7}-\mathrm{H}\right), 2.09 \quad(\mathrm{~s}, 3 \mathrm{H}$, $\left.\mathrm{C}_{17} \mathrm{OOCCH}_{3}\right), 1.95\left(\mathrm{dd}, J=13.4,7.0 \mathrm{~Hz}, 1 \mathrm{H}\right.$, one of $\left.\mathrm{C}_{8}-\mathrm{H}\right)$, $1.78 \sim 1.42\left(\mathrm{~m}, 10 \mathrm{H}\right.$, one of $\mathrm{C}_{8}-\mathrm{H}$, one of $\mathrm{C}_{10}-\mathrm{H}, \mathrm{C}_{18}-\mathrm{H}$, $\left.\mathrm{C}_{19}-\mathrm{H}, \mathrm{C}_{20}-\mathrm{H}, \mathrm{C}_{22}-\mathrm{H}\right), 1.31 \sim 1.29\left(\mathrm{~m}, 3 \mathrm{H}, \mathrm{C}_{6}-\mathrm{H}\right), 1.15$ (dd, $\left.J=16,7.5 \mathrm{~Hz}, 3 \mathrm{H}, \mathrm{C}_{16}-\mathrm{CH}_{3}\right), 1.02 \sim 0.98\left(\mathrm{~m}, 1 \mathrm{H}, \mathrm{C}_{11}-\mathrm{H}\right)$, 0.84 (t, $\left.J=7.5 \mathrm{~Hz}, 3 \mathrm{H}, \mathrm{C}_{23}-\mathrm{H}\right) ;{ }^{13} \mathrm{C} \mathrm{NMR}(100 \mathrm{MHz}$, $\left.\mathrm{CDCl}_{3}\right) \delta: 201.60,172.78,170.93,147.67,144.62,129.31$, $128.70,95.59,82.70,81.83,77.41,77.00,75.53,68.04$, $59.39,58.07,49.93,48.06,46.41,45.51,41.56,36.74$, $32.93,30.63,28.59,21.44,18.20,16.24$, 9.70; FT-MS (ESI) calcd for $\mathrm{C}_{35} \mathrm{H}_{56} \mathrm{NO}_{10}\left[\mathrm{M}+\mathrm{NH}_{4}\right]^{+}$650.38987, found 650.39004 .

3.2.2 (2R,3aS, 5aR, $5 b S, 9 S, 13 S, 14 R, 16 a S, 16 b R)-9-乙$ 基-14-甲基-7,15-二羰基-2-(( (2R,3R,4R,5S,6S)-3,4,5-三 甲氧基-6-甲基四氢- $2 H$-吡喃-2-)氧代)- $2,3,3 a, 5 a, 5 b, 6$, $7,9,10,11,12,13,14,15,16 a, 16 b$-十六氢- $1 H$ - $a s$-引达省并 $[3,2-d][1]$ 氧杂十二元环-13-环己羧酸酯 $(3 \mathbf{b})$ 的合成

溶剂换为无水二氯甲烷 $(10 \mathrm{~mL})$, 酰化试剂改为环 己基甲酰氯 $(0.36 \mathrm{~g}, 2.5 \mathrm{mmol})$. 柱层析的展开剂为 $V$ (石 油醚 $): V($ 乙酸乙酯 $)=6: 1 \quad\left(R_{\mathrm{f}}=0.2\right)$. 旋干溶剂得黄色 油状液体. 其它同上. ${ }^{1} \mathrm{H}$ NMR $\left(400 \mathrm{MHz}, \mathrm{CDCl}_{3}\right) \delta: 6.79$ $\left(\mathrm{s}, 1 \mathrm{H}, \mathrm{C}_{13}-\mathrm{H}\right), 5.89 \sim 5.87\left(\mathrm{~m}, 1 \mathrm{H}, \mathrm{C}_{6}-\mathrm{H}\right), 5.81 \sim 5.78(\mathrm{~m}$, $\left.1 \mathrm{H}, \mathrm{C}_{5}-\mathrm{H}\right), 4.85\left(\mathrm{~s}, 1 \mathrm{H}, \mathrm{C}_{1^{\prime}}-\mathrm{H}\right), 4.72 \sim 4.63\left(\mathrm{~m}, 1 \mathrm{H}, \mathrm{C}_{21}-\mathrm{H}\right)$, $4.34 \sim 4.29\left(\mathrm{~m}, 1 \mathrm{H}, \mathrm{C}_{9}-\mathrm{H}\right), 3.67 \sim 3.61\left(\mathrm{~m}, 1 \mathrm{H}, \mathrm{C}_{17}-\mathrm{H}\right)$, $3.61 \sim 3.58\left(\mathrm{~m}, 1 \mathrm{H}, \mathrm{C}_{2^{\prime}}-\mathrm{H}\right), 3.56\left(\mathrm{~s}, 3 \mathrm{H}, \mathrm{C}_{4^{\prime}}-\mathrm{OCH}_{3}\right), 3.51(\mathrm{~s}$, $\left.3 \mathrm{H}, \mathrm{C}_{2^{\prime}}-\mathrm{OCH}_{3}\right), 3.50\left(\mathrm{~s}, 3 \mathrm{H}, \mathrm{C}_{3^{\prime}}-\mathrm{OCH}_{3}\right), 3.50 \sim 3.45(\mathrm{~m}$, $\left.2 \mathrm{H}, \mathrm{C}_{4}-\mathrm{H}, \mathrm{C}_{5^{\prime}}-\mathrm{H}\right), 3.39$ (dd, J=9.4, $\left.3.3 \mathrm{~Hz}, 1 \mathrm{H}, \mathrm{C}_{3^{\prime}}-\mathrm{H}\right)$, $3.18 \sim 3.14\left(\mathrm{~m}, 1 \mathrm{H}, \mathrm{C}_{16}-\mathrm{H}\right), 3.12 \sim 3.09(\mathrm{~m}, 2 \mathrm{H}$, one of $\left.\mathrm{C}_{2}-\mathrm{H}, \mathrm{C}_{4^{\prime}}-\mathrm{H}\right), 3.07 \sim 3.02\left(\mathrm{~m}, 1 \mathrm{H}, \mathrm{C}_{3}-\mathrm{H}\right), 2.91 \sim 2.89(\mathrm{~m}$, $\left.1 \mathrm{H}, \mathrm{C}_{12}-\mathrm{H}\right), 2.43\left(\mathrm{dd}, J=8.0,2.0 \mathrm{~Hz}, 1 \mathrm{H}\right.$, one of $\left.\mathrm{C}_{2}-\mathrm{H}\right)$, $2.34 \sim 2.13\left(\mathrm{~m}, 3 \mathrm{H}\right.$, one of $\left.\mathrm{C}_{10}-\mathrm{H}, \mathrm{C}_{7}-\mathrm{H}, \mathrm{C}_{17} \mathrm{OOCC}-\mathrm{H}\right)$, $1.92\left(\mathrm{dd}, J=13.4,7.0 \mathrm{~Hz}, 1 \mathrm{H}\right.$, one of $\left.\mathrm{C}_{8}-\mathrm{H}\right), 1.78 \sim 1.41$ (m, $15 \mathrm{H}$, one of $\mathrm{C}_{8}-\mathrm{H}$, one of $\mathrm{C}_{10}-\mathrm{H}, \mathrm{C}_{18}-\mathrm{H}, \mathrm{C}_{19}-\mathrm{H}, \mathrm{C}_{20}-\mathrm{H}$, $\left.\mathrm{C}_{22}-\mathrm{H}, \mathrm{C}_{6} \mathrm{H}_{5}\right), 1.31 \sim 1.29\left(\mathrm{~m}, 3 \mathrm{H}, \mathrm{C}_{6},-\mathrm{H}\right), 1.12(\mathrm{dd}, J=16$, $\left.7.8 \mathrm{~Hz}, 3 \mathrm{H}, \mathrm{C}_{16}-\mathrm{CH}_{3}\right), 0.96 \sim 0.88\left(\mathrm{~m}, 1 \mathrm{H}, \mathrm{C}_{11}-\mathrm{H}\right), 0.84(\mathrm{t}$,
$\left.J=7.5 \mathrm{~Hz}, 3 \mathrm{H}, \mathrm{C}_{23}-\mathrm{H}\right) ;{ }^{13} \mathrm{C} \mathrm{NMR}\left(100 \mathrm{MHz}, \mathrm{CDCl}_{3}\right) \delta$ : 201.14, 175.22, 172.34, 147.13, 143.89, 129.16, 128.59, $95.34,82.09,80.88,77.49,76.49,76.24,74.33,67.75$, $60.78,58.82,57.48,47.46,45.90,45.33,43.28,41.33$, $40.96,37.25,34.01,32.50,28.00,25.26,20.88,17.62$, 16.21, 9.21, 8.88; FT-MS (ESI) calcd for $\mathrm{C}_{40} \mathrm{H}_{61} \mathrm{O}_{10}[\mathrm{M}+$ $\mathrm{H}^{+}$701.42592, found 701.42718 .

3.2.3 (2R,3aS, 5aR,5bS,9S,13S,14R,16aS,16bR)-9-乙 基-14-甲基-7,15-二羰基-2-(( $(2 R, 3 R, 4 R, 5 S, 6 S)-3,4,5$-三 甲氧基-6-甲基四氢- $2 H$-吡喃-2-)氧代)-2,3,3a,5a,5b,6, $7,9,10,11,12,13,14,15,16 a, 16 b$-十六氢- $1 H$ - $a s$-引达省并 [3,2-d][1]氧杂十二元环-13-糠酸酯 $(3 \mathrm{c})$ 的合成

酰化试剂改为 2 -四氢糠酰氯 $(0.25 \mathrm{~mL} 2.5 \mathrm{mmol})$, 柱层析的展开剂为 $V$ (石油醚) : $V$ (乙酸乙酯 $)=3: 1$ $\left(R_{\mathrm{f}}=0.2\right)$, 旋干溶剂得黄色油状液体. 其它同上. ${ }^{1} \mathrm{H}$ NMR (400 MHz, $\left.\mathrm{CDCl}_{3}\right) \delta: 6.77\left(\mathrm{~s}, 1 \mathrm{H}, \mathrm{C}_{13}-\mathrm{H}\right), 5.88 \sim$ $5.85\left(\mathrm{~m}, 1 \mathrm{H}, \mathrm{C}_{6}-\mathrm{H}\right), 5.79 \sim 5.76\left(\mathrm{~m}, 1 \mathrm{H}, \mathrm{C}_{5}-\mathrm{H}\right), 4.83(\mathrm{~s}$, $\left.1 \mathrm{H}, \mathrm{C}_{1}-\mathrm{H}\right), 4.71 \sim 4.60\left(\mathrm{~m}, 1 \mathrm{H}, \mathrm{C}_{21}-\mathrm{H}\right), 4.46 \sim 4.29(\mathrm{~m}$, $\left.1 \mathrm{H}, \mathrm{C}_{9}-\mathrm{H}\right), 4.03 \sim 3.90\left(\mathrm{~m}, 1 \mathrm{H}, \mathrm{C}_{17}-\mathrm{H}\right), 3.66 \sim 3.59(\mathrm{~m}, 1 \mathrm{H}$,

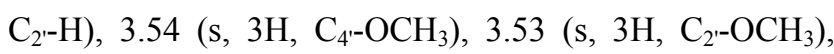
$3.48\left(\mathrm{~s}, 3 \mathrm{H}, \mathrm{C}_{3^{\prime}}-\mathrm{OCH}_{3}\right), 3.46 \sim 3.43\left(\mathrm{~m}, 2 \mathrm{H}, \mathrm{C}_{4}-\mathrm{H}, \mathrm{C}_{5^{\prime}}-\mathrm{H}\right)$, $3.42\left(\mathrm{dd}, J=9.4,3.3 \mathrm{~Hz}, 1 \mathrm{H}, \mathrm{C}_{3^{\prime}}-\mathrm{H}\right), 3.18 \sim 3.13(\mathrm{~m}, 1 \mathrm{H}$, $\left.\mathrm{C}_{16}-\mathrm{H}\right), 3.12 \sim 3.10\left(\mathrm{~m}, 2 \mathrm{H}\right.$, one of $\left.\mathrm{C}_{2}-\mathrm{H}, \mathrm{C}_{4}-\mathrm{H}\right), 3.08 \sim$ $3.02\left(\mathrm{~m}, 1 \mathrm{H}, \mathrm{C}_{3}-\mathrm{H}\right), 2.92 \sim 2.82\left(\mathrm{~m}, 1 \mathrm{H}, \mathrm{C}_{12}-\mathrm{H}\right), 2.42(\mathrm{dd}$, $J=8.0,2.0 \mathrm{~Hz}, 1 \mathrm{H}$, one of $\left.\mathrm{C}_{2}-\mathrm{H}\right), 2.31 \sim 2.19(\mathrm{~m}, 1 \mathrm{H}$, one of $\left.\mathrm{C}_{10}-\mathrm{H}\right), 2.19 \sim 2.12\left(\mathrm{~m}, 1 \mathrm{H}, \mathrm{C}_{7}-\mathrm{H}\right), 2.01 \sim 1.88(\mathrm{~m}, 2 \mathrm{H}$, $\mathrm{C}_{17} \mathrm{OOCCHOCH}{ }_{2} \mathrm{CH}_{2} \mathrm{C}-\mathrm{H}$, one of $\left.\mathrm{C}_{8}-\mathrm{H}\right), 1.78 \sim 1.42(\mathrm{~m}$, $12 \mathrm{H}$, one of $\mathrm{C}_{8}-\mathrm{H}$, one of $\mathrm{C}_{10}-\mathrm{H}, \mathrm{C}_{18}-\mathrm{H}, \mathrm{C}_{19}-\mathrm{H}, \mathrm{C}_{20}-\mathrm{H}$, $\left.\mathrm{C}_{22}-\mathrm{H}, \mathrm{C}_{17} \mathrm{OOCCHC}-\mathrm{H}, \mathrm{C}_{17} \mathrm{OOCCHOCH}_{2} \mathrm{C}-\mathrm{H}\right), 1.28 \sim$ $1.26\left(\mathrm{~m}, 3 \mathrm{H}, \mathrm{C}_{6^{\prime}} \mathrm{H}\right), 1.12(\mathrm{dd}, J=16.0,8.4 \mathrm{~Hz}, 3 \mathrm{H}$, $\left.\mathrm{C}_{16}-\mathrm{CH}_{3}\right), 0.93 \sim 0.84\left(\mathrm{~m}, 1 \mathrm{H}, \mathrm{C}_{11}-\mathrm{H}\right), 0.79(\mathrm{t}, J=7.5 \mathrm{~Hz}$, $\left.3 \mathrm{H}, \mathrm{C}_{23}-\mathrm{H}\right) ;{ }^{13} \mathrm{C}$ NMR $\left(100 \mathrm{MHz}, \mathrm{CDCl}_{3}\right) \delta: 200.91$, $172.76,172.40,147.27,143.95,129.52,128.57,95.54$, $82.40,81.12,77.47,76.84,75.37,69.45,68.07,61.18$, $60.90,59.04,57.50,49.55,47.67,46.17,45.53,41.26$, $36.44,34.31,32.66,28.08,20.75,17.91,9.47$; FT-MS (ESI) calcd for $\mathrm{C}_{38} \mathrm{H}_{57} \mathrm{O}_{11}[\mathrm{M}+\mathrm{H}]^{+}$689.38954, found 689.39130 .

3.2.4 (2R,3aS, $5 a R, 5 b S, 9 S, 13 S, 14 R, 16 a S, 16 b R)-9-$ 乙 基-14-甲基-7, 15-二羰基-2-(( $(2 R, 3 R, 4 R, 5 S, 6 S)-3,4,5$-三 甲氧基-6-甲基四氢- $2 H$-吡喃-2-)氧代)-2,3,3a,5a,5b,6, $7,9,10,11,12,13,14,15,16 a, 16 b$-十六氢- $1 H$ - $a s$-引达省并 $[3,2-d][1]$ 氧杂十二元环-13-烟酸酯 $(3 d)$ 的合成

加料顺序改为: 氩气保护下, 将少量无水二氯甲烷 和烟酰氯(0.354 g $2.5 \mathrm{mmol})$ 加入 $100 \mathrm{~mL}$ 两口瓶中, 再 
加入多杀菌素降解产物 I (0.3 g, $0.5 \mathrm{mmol})$, DMAP (0.305 g, $2.5 \mathrm{mmol}), \mathrm{DCC}(0.515 \mathrm{~g}, 2.5 \mathrm{mmol})$ 的无水二氯 甲烷 $(10 \mathrm{~mL})$ 溶液. 柱层析的展开剂为 $V$ (石油醚) $: V($ 乙 酸乙酯 $)=1: 1\left(R_{\mathrm{f}}=0.2\right)$, 旋干溶剂得到浅黄色固体. 其它同上. m.p. $105{ }^{\circ} \mathrm{C} ;{ }^{1} \mathrm{H}$ NMR $\left(500 \mathrm{MHz}, \mathrm{CDCl}_{3}\right) \delta$ : $9.23\left(\mathrm{~s}, \quad 1 \mathrm{H}, \quad \mathrm{C}_{17} \mathrm{OOCCC}-\mathrm{H}\right), 8.78 \sim 8.66(\mathrm{~m}, \quad 1 \mathrm{H}$, $\left.\mathrm{C}_{17} \mathrm{OOCCCHNC}-\mathrm{H}\right), 8.31 \sim 8.32\left(\mathrm{~m}, 1 \mathrm{H}, \mathrm{C}_{17} \mathrm{OOCCCHN}-\right.$ CHCHC-H), $7.86 \sim 7.32\left(\mathrm{~m}, 1 \mathrm{H}, \mathrm{C}_{17} \mathrm{OOCCCHNCHC}-\mathrm{H}\right)$, $6.84\left(\mathrm{~s}, 1 \mathrm{H}, \mathrm{C}_{13}-\mathrm{H}\right), 6.31 \sim 6.35\left(\mathrm{~m}, 1 \mathrm{H}, \mathrm{C}_{6}-\mathrm{H}\right), 5.36 \sim 5.30$ $\left(\mathrm{m}, 1 \mathrm{H}, \mathrm{C}_{5}-\mathrm{H}\right), 4.85\left(\mathrm{~s}, 1 \mathrm{H}, \mathrm{C}_{1^{\prime}}-\mathrm{H}\right), 4.71 \sim 4.70(\mathrm{~m}, 1 \mathrm{H}$, $\left.\mathrm{C}_{21}-\mathrm{H}\right), 4.37 \sim 4.30\left(\mathrm{~m}, 1 \mathrm{H}, \mathrm{C}_{9}-\mathrm{H}\right), 4.15 \sim 4.11(\mathrm{~m}, 1 \mathrm{H}$,

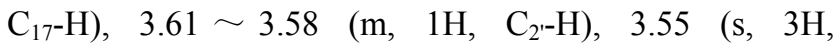
$\left.\mathrm{C}_{4^{\prime}}-\mathrm{OCH}_{3}\right), 3.50\left(\mathrm{~s}, 3 \mathrm{H}, \mathrm{C}_{2^{\prime}}-\mathrm{OCH}_{3}\right), 3.49$ (s, 3H, $\left.\mathrm{C}_{3^{\prime}}-\mathrm{OCH}_{3}\right)$, $3.47 \sim 3.45\left(\mathrm{~m}, 3 \mathrm{H}, \mathrm{C}_{4}-\mathrm{H}, \mathrm{C}_{5^{\prime}}-\mathrm{H}, \mathrm{C}_{3^{\prime}}-\mathrm{H}\right), 3.18 \sim 3.13(\mathrm{~m}$, $\left.1 \mathrm{H}, \mathrm{C}_{16}-\mathrm{H}\right), 3.11 \sim 3.09\left(\mathrm{~m}, 2 \mathrm{H}\right.$, one of $\left.\mathrm{C}_{2}-\mathrm{H}, \mathrm{C}_{4^{-}}-\mathrm{H}\right)$, $3.08 \sim 3.05\left(\mathrm{~m}, 1 \mathrm{H}, \mathrm{C}_{3}-\mathrm{H}\right), 2.94 \sim 2.86\left(\mathrm{~m}, 1 \mathrm{H}, \mathrm{C}_{12}-\mathrm{H}\right)$, $2.42\left(\mathrm{dd}, J=8.0,2.0 \mathrm{~Hz}, 1 \mathrm{H}\right.$, one of $\left.\mathrm{C}_{2}-\mathrm{H}\right), 2.30 \sim 2.24(\mathrm{~m}$, $1 \mathrm{H}$, one of $\left.\mathrm{C}_{10}-\mathrm{H}\right), 2.16 \sim 1.49\left(\mathrm{~m}, 16 \mathrm{H}, \mathrm{C}_{7}-\mathrm{H}, \mathrm{C}_{8}-\mathrm{H}\right.$, one of $\left.\mathrm{C}_{10}-\mathrm{H}, \mathrm{C}_{18}-\mathrm{H}, \mathrm{C}_{19}-\mathrm{H}, \mathrm{C}_{20}-\mathrm{H}, \mathrm{C}_{22}-\mathrm{H}, \mathrm{C}_{6}-\mathrm{H}\right), 1.26$ (dd, $J=$ $\left.16.0,8.5 \mathrm{~Hz}, 3 \mathrm{H}, \mathrm{C}_{16}-\mathrm{CH}_{3}\right), 1.07 \sim 1.01\left(\mathrm{~m}, 1 \mathrm{H}, \mathrm{C}_{11}-\mathrm{H}\right)$, $0.83\left(\mathrm{t}, J=7.5 \mathrm{~Hz}, 3 \mathrm{H}, \mathrm{C}_{23}-\mathrm{H}\right) ;{ }^{13} \mathrm{C}$ NMR $(500 \mathrm{MHz}$, $\left.\mathrm{CDCl}_{3}\right) \delta:$ 201.03, 172.51, 168.10, 164.72, 153.72, 153.65, $153.49,151.42,150.83,147.87,147.70,144.02,137.17$, $134.36,132.81,129.41,128.71,126.16,123.41,123.16$, $95.49,82.26,81.03,77.66,76.45,76.14,67.93,60.99$, $59.02,57.68,56.87,49.87,49.56,47.65,46.07,45.56$, $41.50,41.14,37.43,36.33,34.18,32.65,32.31,30.95$, $30.78,30.13,28.17,26.09,25.25,25.19,24.55,21.06$, 17.81, 16.51, 9.40; FT-MS (ESI) calcd for $\mathrm{C}_{39} \mathrm{H}_{54} \mathrm{NO}_{10}$ $[\mathrm{M}+\mathrm{H}]^{+} \quad 696.37422$, found 696.37250 .

3.2.5 (2R,3aS, $5 a R, 5 b S, 9 S, 13 S, 14 R, 16 a S, 16 b R)-9$ - 乙 基-14-甲基-7,15-二羰基-2-(( $(2 R, 3 R, 4 R, 5 S, 6 S)-3,4,5$-三 甲氧基-6-甲基四氢- $2 \mathrm{H}$-吡喃-2-)氧代)-2,3,3a,5a,5b,6, $7,9,10,11,12,13,14,15,16 a, 16 b$-十六氢- $1 H$ - $a s$-引达省并 $[3,2-d][1]$ 氧杂十二元环-13-金刚烷甲酸酯(3e)的合成

酰化试剂改为金刚烷酰氯 $(0.5 \mathrm{~g}, 2.5 \mathrm{mmol})$, 柱层析 的展开剂为 $V$ (石油醚)：V(乙酸乙酯 $)=5: 1\left(R_{\mathrm{f}}=0.2\right)$, 旋干溶剂得黄色油状液体. 其它同第 3.2.2 节. ${ }^{1} \mathrm{H}$ NMR $\left(400 \mathrm{MHz}, \mathrm{CDCl}_{3}\right) \delta: 6.74\left(\mathrm{~s}, 1 \mathrm{H}, \mathrm{C}_{13}-\mathrm{H}\right), 5.82 \sim 5.79(\mathrm{~m}$, $\left.1 \mathrm{H}, \mathrm{C}_{6}-\mathrm{H}\right), 5.73 \sim 5.71\left(\mathrm{~m}, 1 \mathrm{H}, \mathrm{C}_{5}-\mathrm{H}\right), 4.90 \sim 4.88(\mathrm{~s}, 1 \mathrm{H}$,

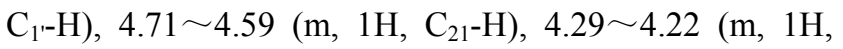
$\left.\mathrm{C}_{9}-\mathrm{H}\right), 4.07 \sim 3.98\left(\mathrm{~m}, 1 \mathrm{H}, \mathrm{C}_{17}-\mathrm{H}\right), 3.61 \sim 3.57(\mathrm{~m}, 1 \mathrm{H}$, $\left.\mathrm{C}_{2^{\prime}}-\mathrm{H}\right), 3.56$ (s, $\left.3 \mathrm{H}, \mathrm{C}_{4^{\prime}}-\mathrm{OCH}_{3}\right), 3.48\left(\mathrm{~s}, 3 \mathrm{H}, \mathrm{C}_{2^{\prime}}-\mathrm{OCH}_{3}\right), 3.45$ (s, 3H, $\mathrm{C}_{3^{\prime}}-\mathrm{OCH}_{3}$ ), $3.41 \sim 3.37$ (m, $2 \mathrm{H}, \mathrm{C}_{4}-\mathrm{H}, \mathrm{C}_{5^{\prime}}-\mathrm{H}$ ), 3.35 $\left(\mathrm{dd}, J=9.4,3.3 \mathrm{~Hz}, 1 \mathrm{H}, \mathrm{C}_{3^{\prime}-\mathrm{H}}\right), 3.13 \sim 3.09(\mathrm{~m}, 1 \mathrm{H}$,
$\left.\mathrm{C}_{16}-\mathrm{H}\right), 3.09 \sim 3.04\left(\mathrm{~m}, 2 \mathrm{H}\right.$, one of $\left.\mathrm{C}_{2}-\mathrm{H}, \mathrm{C}_{4}-\mathrm{H}\right), 3.01 \sim$ $2.94\left(\mathrm{~m}, 1 \mathrm{H}, \mathrm{C}_{3}-\mathrm{H}\right), 2.81 \sim 2.78\left(\mathrm{~m}, 1 \mathrm{H}, \mathrm{C}_{12}-\mathrm{H}\right), 2.36(\mathrm{dd}$, $J=13.6,3.2 \mathrm{~Hz}, 1 \mathrm{H}$, one of $\left.\mathrm{C}_{2}-\mathrm{H}\right), 2.24 \sim 2.18(\mathrm{~m}, 1 \mathrm{H}$, one of $\left.\mathrm{C}_{10}-\mathrm{H}\right), 2.10 \sim 2.08\left(\mathrm{~m}, 1 \mathrm{H}, \mathrm{C}_{7}-\mathrm{H}\right), 2.04 \sim 1.54(\mathrm{~m}, 26 \mathrm{H}$, $\mathrm{C}_{8}-\mathrm{H}$, one of $\mathrm{C}_{10}-\mathrm{H}, \mathrm{C}_{18}-\mathrm{H}, \mathrm{C}_{19}-\mathrm{H}, \mathrm{C}_{20}-\mathrm{H}, \mathrm{C}_{22}-\mathrm{H}$, adamantane-15H), 1.18 (d, $\left.J=6.3 \mathrm{~Hz}, 3 \mathrm{H}, \mathrm{C}_{6^{\prime}}-\mathrm{H}\right), 1.13(\mathrm{dd}, J=6.8$ $\left.\mathrm{Hz}, 3 \mathrm{H}, \mathrm{C}_{16}-\mathrm{CH}_{3}\right), 1.11 \sim 0.98\left(\mathrm{~m}, 1 \mathrm{H}, \mathrm{C}_{11}-\mathrm{H}\right), 0.75(\mathrm{t}, J=$ $\left.7.5 \mathrm{~Hz}, 3 \mathrm{H}, \mathrm{C}_{23}-\mathrm{H}\right) ;{ }^{13} \mathrm{C}$ NMR $\left(100 \mathrm{MHz}, \mathrm{CDCl}_{3}\right) \delta$ : $201.13,178.03,172.68,147.39,144.26,129.32,128.24$, $95.66,82.30,80.73,77.41,74.90,74.15,67.97,60.86$, $59.93,58.96,57.61,51.44,45.69,40.52,38.89,38.79$, $36.56,32.51,28.15,27.98,21.04,17.19,14.20,9.37$; FT-MS (ESI) calcd for $\mathrm{C}_{37} \mathrm{H}_{65} \mathrm{~N}_{7} \mathrm{O}_{10} 767.47874$, found 767.47691 .

3.2.6 (2R,3aS, $5 a R, 5 b S, 9 S, 13 S, 14 R, 16 a S, 16 b R)-9$ - 乙 基-14- 甲基-7,15-二羰基-2,3,3a,5a,5b,6,7,9,10,11,12， $13,14,15,16 a, 16 b$-十六氢- $1 H$ - $a s$-引达省并 [3,2- $d][1]$ 氧 杂十二元环-2,13-二乙酸酯(3f)的合成

氩气保护下, 多杀菌素降解产物 II $(0.2 \mathrm{~g}, 0.5$ mmol), DMAP (0.6 g, $5 \mathrm{mmol}$ )依次加入到无水二氯甲烷 $(10 \mathrm{~mL})$ 中并搅拌至全部溶解, 用注射器缓慢加入乙酸 酐( $0.5 \mathrm{~mL}, 5 \mathrm{mmol})$, 回流反应 $24 \mathrm{~h}, \mathrm{TLC}$ 监测反应. 反 应结束后依次用 $5 \% \mathrm{NaHCO}_{3}$ 溶液、饱和 $\mathrm{NaCl}$ 溶液洗涤, 合并有机相, 有机相用无水 $\mathrm{Na}_{2} \mathrm{SO}_{4}$ 干燥, 柱层析分离, 展开剂为 $V$ (石油醚) $: V($ 乙酸乙酯 $)=4: 1 \quad\left(R_{\mathrm{f}}=0.3\right)$, 旋 干溶剂得到白色固体. m.p. $95{ }^{\circ} \mathrm{C} ;{ }^{1} \mathrm{H}$ NMR $(500 \mathrm{MHz}$, $\left.\mathrm{CDCl}_{3}\right) \delta: 6.77\left(\mathrm{~s}, 1 \mathrm{H}, \mathrm{C}_{13}-\mathrm{H}\right), 5.91 \sim 5.88\left(\mathrm{~m}, 1 \mathrm{H}, \mathrm{C}_{6}-\mathrm{H}\right)$, $5.81 \sim 5.79\left(\mathrm{~m}, 1 \mathrm{H}, \mathrm{C}_{5}-\mathrm{H}\right), 5.19 \sim 5.13\left(\mathrm{~m}, 1 \mathrm{H}, \mathrm{C}_{21}-\mathrm{H}\right)$, $5.03 \sim 4.94\left(\mathrm{~m}, 1 \mathrm{H}, \mathrm{C}_{9}-\mathrm{H}\right), 4.71 \sim 4.68\left(\mathrm{~m}, 1 \mathrm{H}, \mathrm{C}_{17}-\mathrm{H}\right)$, $3.50 \sim 3.48\left(\mathrm{~m}, 1 \mathrm{H}, \mathrm{C}_{4}-\mathrm{H}\right), 3.40 \sim 3.32\left(\mathrm{~m}, 1 \mathrm{H}, \mathrm{C}_{16}-\mathrm{H}\right)$, $3.12\left(\mathrm{dd}, J=15.0,2.5 \mathrm{~Hz}, 1 \mathrm{H}\right.$, one of $\left.\mathrm{C}_{2}-\mathrm{H}\right), 3.07 \sim 3.02$ $\left(\mathrm{m}, 1 \mathrm{H}, \mathrm{C}_{3}-\mathrm{H}\right), 2.92 \sim 2.86\left(\mathrm{~m}, 1 \mathrm{H}, \mathrm{C}_{12}-\mathrm{H}\right), 2.43(\mathrm{dd}, J=$ 13.6, $2.5 \mathrm{~Hz}, 1 \mathrm{H}$, one of $\left.\mathrm{C}_{2}-\mathrm{H}\right), 2.24 \sim 2.18(\mathrm{~m}, 1 \mathrm{H}$, one of $\left.\mathrm{C}_{10}-\mathrm{H}\right), 2.18 \sim 2.16\left(\mathrm{~m}, 1 \mathrm{H}, \mathrm{C}_{7}-\mathrm{H}\right), 2.05(\mathrm{~s}, 3 \mathrm{H}$, $\left.\mathrm{C}_{17} \mathrm{OOCCH}_{3}\right), 2.02\left(\mathrm{~s}, 3 \mathrm{H}, \mathrm{C}_{9} \mathrm{OOCCH}_{3}\right), 1.98 \sim 1.94(\mathrm{~m}$, $1 \mathrm{H}$, one of $\left.\mathrm{C}_{8}-\mathrm{H}\right), 1.73 \sim 1.42\left(\mathrm{~m}, 9 \mathrm{H}\right.$, one of $\mathrm{C}_{8}-\mathrm{H}, \mathrm{C}_{19}-\mathrm{H}$, $\left.\mathrm{C}_{20}-\mathrm{H}, \mathrm{C}_{22}-\mathrm{H}\right), 1.32 \sim 1.23\left(\mathrm{~m}, 2 \mathrm{H}\right.$, one of $\left.\mathrm{C}_{10}-\mathrm{H}, \mathrm{C}_{19}-\mathrm{H}\right)$, $1.23 \sim 1.20\left(\mathrm{~m}, 2 \mathrm{H}\right.$, one of $\mathrm{C}_{10}-\mathrm{H}$, one of $\left.\mathrm{C}_{19}-\mathrm{H}\right), 1.11(\mathrm{~d}$, $\left.J=10.0 \mathrm{~Hz}, 3 \mathrm{H}, \mathrm{C}_{16}-\mathrm{CH}_{3}\right), 1.02 \sim 0.98\left(\mathrm{~m}, 1 \mathrm{H}, \mathrm{C}_{11}-\mathrm{H}\right)$, $0.80\left(\mathrm{t}, J=10.0 \mathrm{~Hz}, 3 \mathrm{H}, \mathrm{C}_{23}-\mathrm{H}\right) ;{ }^{13} \mathrm{C}$ NMR $(500 \mathrm{MHz}$, $\left.\mathrm{CDCl}_{3}\right) \delta: 201.19,172.52,170.82,170.47,147.06,144.17$, $128.98,128.91,77.34,77.29,77.09,76.84,75.05,49.46$, $47.59,46.27,45.45,41.53,41.34,37.17,37.06,34.14$, $32.51,30.12,28.18,21.29,21.05,16.29,9.32$; FT-MS (ESI) calcd for $\mathrm{C}_{28} \mathrm{H}_{39} \mathrm{O}_{7}[\mathrm{M}+\mathrm{H}]^{+}$487.26903, found 
487.26815 .

\subsection{7 (2R,3aS, $5 a R, 5 b S, 9 S, 13 S, 14 R, 16 a S, 16 b R)-9$ - 乙} 基-14-甲基-7,15-二羰基-2,3,3a,5a,5b,6,7,9,10,11,12， $13,14,15,16 a, 16 b$-十六氢- $1 H$ - $a s$-引达省并 [3,2- $d][1]$ 氧 杂十二元环-2,13-二三氟乙酸酯 $(3 \mathrm{~g})$ 的合成

酰化试剂改为三氟乙酸酩 $(0.8 \mathrm{~mL} 5 \mathrm{mmol})$, 柱层析 的展开剂为 $V$ (石油醚) $: V($ 乙酸乙酯 $)=3: 1\left(R_{\mathrm{f}}=0.9\right)$, 旋干溶剂得到黄色油状液体. 其它同上. ${ }^{1} \mathrm{H}$ NMR $(400$ $\left.\mathrm{MHz}, \mathrm{CDCl}_{3}\right) \delta: 6.79\left(\mathrm{~s}, 1 \mathrm{H}, \mathrm{C}_{13}-\mathrm{H}\right), 5.91 \sim 5.89(\mathrm{~m}, 1 \mathrm{H}$, $\left.\mathrm{C}_{6}-\mathrm{H}\right), 5.86 \sim 5.83\left(\mathrm{~m}, 1 \mathrm{H}, \mathrm{C}_{5}-\mathrm{H}\right), 5.41 \sim 5.37(\mathrm{~m}, 1 \mathrm{H}$, $\left.\mathrm{C}_{21}-\mathrm{H}\right), 5.22 \sim 5.17\left(\mathrm{~m}, 1 \mathrm{H}, \mathrm{C}_{9}-\mathrm{H}\right), 4.73 \sim 4.69(\mathrm{~m}, 1 \mathrm{H}$, $\left.\mathrm{C}_{17}-\mathrm{H}\right), 4.34 \sim 4.29\left(\mathrm{~m}, 1 \mathrm{H}, \mathrm{C}_{4}-\mathrm{H}\right), 3.56 \sim 3.49(\mathrm{~m}, 1 \mathrm{H}$, $\left.\mathrm{C}_{16}-\mathrm{H}\right), 3.13\left(\mathrm{dd}, J=15.0,2.5 \mathrm{~Hz}, 1 \mathrm{H}\right.$, one of $\left.\mathrm{C}_{2}-\mathrm{H}\right)$, $3.08 \sim 3.05\left(\mathrm{~m}, 1 \mathrm{H}, \mathrm{C}_{3}-\mathrm{H}\right), 2.99 \sim 2.92\left(\mathrm{~m}, 1 \mathrm{H}, \mathrm{C}_{12}-\mathrm{H}\right)$, $2.57 \sim 2.51\left(\mathrm{dd}, J=16.0,9.0 \mathrm{~Hz}, 1 \mathrm{H}\right.$, one of $\left.\mathrm{C}_{2}-\mathrm{H}\right), 2.46 \sim$ $2.41\left(\mathrm{~m}, 1 \mathrm{H}\right.$, one of $\left.\mathrm{C}_{10}-\mathrm{H}\right), 2.34 \sim 2.24\left(\mathrm{~m}, 1 \mathrm{H}, \mathrm{C}_{7}-\mathrm{H}\right)$, $2.15 \sim 2.09\left(\mathrm{~m}, 1 \mathrm{H}\right.$, one of $\left.\mathrm{C}_{8}-\mathrm{H}\right), 1.71 \sim 1.48(\mathrm{~m}, 6 \mathrm{H}$, $\mathrm{C}_{18}-2 \mathrm{H}$, one of $\left.\mathrm{C}_{8}-\mathrm{H}, \mathrm{C}_{19}-\mathrm{H}, \mathrm{C}_{20}-\mathrm{H}, \mathrm{C}_{22}-\mathrm{H}\right), 1.37 \sim 1.29(\mathrm{~m}$, $2 \mathrm{H}$, one of $\left.\mathrm{C}_{10}-\mathrm{H}, \mathrm{C}_{19}-\mathrm{H}\right), 1.20 \sim 1.17(\mathrm{~m}, 2 \mathrm{H}$, one of $\mathrm{C}_{10}-\mathrm{H}$, one of $\left.\mathrm{C}_{19}-\mathrm{H}\right), 1.06\left(\mathrm{~d}, J=10.0 \mathrm{~Hz}, 3 \mathrm{H}, \mathrm{C}_{16}-\mathrm{CH}_{3}\right)$, $0.98 \sim 0.94\left(\mathrm{~m}, 1 \mathrm{H}, \mathrm{C}_{11}-\mathrm{H}\right), 0.82\left(\mathrm{t}, J=8.0 \mathrm{~Hz}, 3 \mathrm{H}, \mathrm{C}_{23}-\mathrm{H}\right)$; ${ }^{13} \mathrm{C}$ NMR $\left(100 \mathrm{MHz}, \mathrm{CDCl}_{3}\right) \delta: 199.69,173.36,171.94$, $146.73,128.87,127.82,80.05,79.88,76.89,75.95,48.89$, $47.34,45.84,40.71,40.16,36.16,31.74,29.53,27.68$, 20.10, 15.88, 8.93; HRMALDI calcd for $\mathrm{C}_{26} \mathrm{H}_{32} \mathrm{~F}_{6} \mathrm{NO}_{7} \mathrm{Na}$ $[\mathrm{M}+\mathrm{Na}]^{+}$617.194443, found 617.195090.

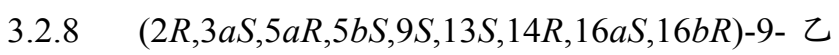

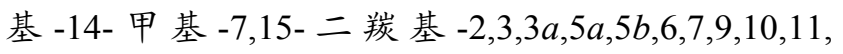
$12,13,14,15,16 a, 16 b$-十六氢- $1 H$ - $a s$-引达省并 $[3,2-d][1]$ 氧杂十二元环-2,13-二金刚烷甲酸酯 $(3 \mathbf{h})$ 的合成

酰化试剂改为金刚烷酰氯(1.0 g, $5 \mathrm{mmol})$, 柱层析 的展开剂为 $V$ (石油醚)：V(乙酸乙酯 $)=1: 1\left(R_{\mathrm{f}}=0.8\right)$, 旋干溶剂得到浅黄色固体. 其它同上. ${ }^{1} \mathrm{H}$ NMR (400 $\left.\mathrm{MHz}, \mathrm{CDCl}_{3}\right) \delta: 6.81\left(\mathrm{~s}, 1 \mathrm{H}, \mathrm{C}_{13}-\mathrm{H}\right), 5.90 \sim 5.88(\mathrm{~m}, 1 \mathrm{H}$, $\left.\mathrm{C}_{6}-\mathrm{H}\right), 5.82 \sim 5.79\left(\mathrm{~m}, 1 \mathrm{H}, \mathrm{C}_{5}-\mathrm{H}\right), 5.17 \sim 5.12(\mathrm{~m}, 1 \mathrm{H}$, $\left.\mathrm{C}_{21}-\mathrm{H}\right), 5.96 \sim 4.96\left(\mathrm{~m}, 1 \mathrm{H}, \mathrm{C}_{9}-\mathrm{H}\right), 4.74 \sim 4.66(\mathrm{~m}, 1 \mathrm{H}$, $\left.\mathrm{C}_{17}-\mathrm{H}\right), 3.67 \sim 3.63\left(\mathrm{~m}, 1 \mathrm{H}, \mathrm{C}_{4}-\mathrm{H}\right), 3.45 \sim 3.39(\mathrm{~m}, 1 \mathrm{H}$, $\left.\mathrm{C}_{16}-\mathrm{H}\right), 3.14\left(\mathrm{dd}, J=15.0,2.5 \mathrm{~Hz}, 1 \mathrm{H}\right.$, one of $\left.\mathrm{C}_{2}-\mathrm{H}\right)$, $3.10 \sim 3.04\left(\mathrm{~m}, 1 \mathrm{H}, \mathrm{C}_{3}-\mathrm{H}\right), 2.92 \sim 2.87\left(\mathrm{~m}, 1 \mathrm{H}, \mathrm{C}_{12}-\mathrm{H}\right)$, $2.44\left(\mathrm{dd}, J=13.6,2.5 \mathrm{~Hz}, 1 \mathrm{H}\right.$, one of $\left.\mathrm{C}_{2}-\mathrm{H}\right), 2.27 \sim 2.18$ $\left(\mathrm{m}, 1 \mathrm{H}\right.$, one of $\left.\mathrm{C}_{10}-\mathrm{H}\right), 2.15 \sim 1.42\left(\mathrm{~m}, 38 \mathrm{H}, \mathrm{C}_{7}-\mathrm{H}, \mathrm{C}_{18}-2 \mathrm{H}\right.$, $\mathrm{C}_{8}-\mathrm{H}, \mathrm{C}_{19}-\mathrm{H}$, one of $\mathrm{C}_{20}-\mathrm{H}, \mathrm{C}_{22}-\mathrm{H}, \mathrm{C}_{9}$-adamantane- $15 \mathrm{H}$, $\mathrm{C}_{17}$-adamantane- $\left.15 \mathrm{H}\right), 1.35 \sim 1.23\left(\mathrm{~m}, 4 \mathrm{H}, \mathrm{C}_{10}-\mathrm{H}, \mathrm{C}_{19}-\mathrm{H}\right)$, $1.10\left(\mathrm{~d}, J=6.0 \mathrm{~Hz}, 3 \mathrm{H}, \mathrm{C}_{16}-\mathrm{CH}_{3}\right), 0.85 \sim 0.79(\mathrm{~m}, 4 \mathrm{H}$, $\left.\mathrm{C}_{11}-\mathrm{H}, \mathrm{C}_{23}-\mathrm{H}\right) ;{ }^{13} \mathrm{C}$ NMR $\left(100 \mathrm{MHz}, \mathrm{CDCl}_{3}\right) \delta: 183.02$,
$179.51,178.92,176.94,146.20,128.34,128.10,76.80$, $76.48,76.17,75.39,73.95,59.43,50.55,48.75,46.73$, $45.60,44.66,38.43,36.96,35.74,33.30,31.25,29.15$, $27.31,27.10,21.87,20.34,15.70,13.65,8.44$; FT-MS (ESI) calcd for $\mathrm{C}_{46} \mathrm{H}_{63} \mathrm{O}_{7}[\mathrm{M}+\mathrm{H}]^{+} 727.45683$, found 727.45492 .

\section{3 杀虫活性研究测试方法描述}

\subsection{1 杀虫活性的测试方法}

采用叶片药膜法 ${ }^{[24]}$. 原药先用少量丙酮溶解, 然后 用 $1 \%$ Triton- 100 稀释至浓度为 $100 \mathrm{mg} / \mathrm{L}, 1 \%$ Triton-100 水为对照, 重复三次, 每次处理 10 头测试虫子; 取新鲜 无污染的菜叶在测试药液中浸泡 $10 \mathrm{~s}$ 后取出, 晾干, 剪 切为适合大小并放置在培养具上, 为防止菜叶变干, 再 次在每个培养基中滴加少量水; 分别接入大小基本一致 的待测虫子; 随后封膜, 膜上扎孔; 室温下放置 $24 \mathrm{~h}$ 或 $48 \mathrm{~h}$ 后, 观察结果并计算杀虫率; 以小毛笔或镊子轻触 虫体, 不能协调运动视为死亡.

\subsection{2 杀菌活性的测试}

采用滤纸片法对所合成的 8 种目标化合物进行杀菌 活性测试, 溶剂丙酮为空白对照组, 将 8 种化合物分别 溶于丙酮配制成 $2000 \mathrm{mg} / \mathrm{L}$ 的溶液, 备用; 取 PDA 在微 波炉中加热, 使其融化, 部分 PDA 直接倒入培养血中静 置冷却, 凝固后在表面均匀涂上细菌培养液, 另一部分 $\mathrm{PDA}$ 与细菌培养液混合均匀后倒入培养血中, 制得两 类细菌样本; 将含药量为 $100 \mu \mathrm{L}$ 的滤纸移至已经加入 细菌培养液的培养血, 对照组的滤纸不浸药; 将细菌置 于 $28{ }^{\circ} \mathrm{C}$ 条件下培养, $48 \mathrm{~h}$ 后观察药物对细菌的抑制作 用.

\section{References}

[1] Yao, X. Y; Zhang, Y. X. Med. Recapitulate 2011, 32(7), 319 (in Chinese). (姚晓英，张永信，医药综述, 2011, 32(7), 319.)

[2] Weissman, K. J. Philos. Trans. R. Soc., A 2004, 362(1825), 2671.

[3] Floss, H. G. J. Ind. Microbiol. Biotechnol. 2001, 27(3), 183.

[4] Putnam, S. D.; Sader, H. S.; Farrell, D. J.; Biedenbach, D. J. Int. J. Antimicrob. Agents 2011, 37(1), 39.

[5] Jiang, L. J.; Or, Y. S. Antimicrob. Agents. Chemother. 2009, 53(8), 3218.

[6] Ge, H.; Shen, S. Y. World Clinical Drugs 2007, 28(6), 376 (in Chinese).

(葛涵, 沈舜义，世界临床药物, 2007, 28(6), 376.)

[7] Lee, H. Y.; Chung, H. S.; Hang, C.; Khosla, C.; Walsh, C. T.; Kahne, D.; Walker, S. J. Am. Chem. Soc. 2004, 126(32), 9924.

[8] Rospide, M. F.; Biedenbach, D. J. Int. J. Antimicrob. Agents 2001, 17(6), 451

[9] Garza, R. G.; Xiong, L.; Zong, P. J. Bacteriol. 2001, 183, 6898.

[10] Farrell, D. J.; Sader, H. S.; Castanheira, M. Int. J. Antimicrob. Agents 2010, 35(6), 537.

[11] Holt, K. M.; Opit, G. P.; Nechols, J. R. Exp. Appl. Acarol. 2006, 38, 
141.

[12] Mota, S. D.; Hollingworth, R. M.; Grafius, E. J. Pest Manage. Sci. 2006, 62, 30 .

[13] Du, S. T.; Zhu, M. J.; Lang, S. Z. Agrochemicals 2005, 44(10), 441 (in Chinese).

(杜顺堂, 朱明军, 梁世中, 农药, 2005, 44(10), 441.)

[14] Thompson, G. D.; Dutton, R.; Sparks, T. C. Pest Manage. Sci. 2000, 56(8), 696.

[15] Ou, X. M.; Liu, S. Y.; Pei, H.; Ma, D. Y.; Li, M.; Liu, L. J.; Wang, X. Z.; Yi, Z. H.; Bai, J. J.; Yu, K. CN 102977166, 2013 [Chem. Abstr. 2013, 158, 495574].

[16] Li, R.; Wang, Q. M.; Huang, R. Q. Chin. J. Pestic. Sci. 2003, 5(2), 1 (in Chinese). (李如, 汪清民, 黄润秋, 农药学学报, 2003, 5(2), 1.)

[17] Thomas, C. Pestic. Biochem. Phys. 2000, 67(3), 187.

[18] Sparks, T. C.; Crouse, G. D.; Dripps, J. E.; Anzeveno, P.; Martynow, J.; Deamicis, V.; Gifford, J. J. Comput.-Aided Mol. Des.
2008, 22, 393.

[19] Waldron, C.; Matsushima, P.; Rosteck, P. R.; Broughton, M. C.; Turner, J.; Madduri, K; Crawdord, K. P.; Merlo, D. J.; Baltz, R. H. J. Chem. Biol. 2001, 8(5), 487.

[20] Deamicis, C. V.; Anzeveno, P. B.; Martynow, J. G.; McLaren, K. L.; Green, F. R., III; Sparks, T. C.; Kirst, H. A.; Creemer, L. C.; Worden, T. V.; Schoonover, J. R., Jr.; Gifford, J. M.; Hatton, C. J.; Hegde, V. B.; Crouse, G. D.; Thoreen, B. R.; Ricks, M. J. US 006001981, 1999 [Chem. Abstr. 1999, 132, 35986].

[21] Podhorez, D. E.; Roth, G. A.; Molzahn, D. C.; Adaway, T. US 2008/0108800, 2008 [Chem. Abstr. 2008, 148, 517925].

[22] Creemer, L. C.; Kirst, H. A.; Paschal, J. W. J. Antibiot. 1998, 51(8), 795.

[23] Wanka, L.; Iqbal, K.; Schreiner, P. R. Chem. Rev. 2013, 113(5), 3516.

[24] Wang, H.; Yang, Z. K.; Fan, Z. J.; Wu, Q. J.; Zhang, Y. J.; Mi, N.; Wang, S. X.; Zhang, Z. C. Agric. Food Chem. 2011, 59, 628.

(Li, L.; Lu, Z.) 